\title{
Evaluation of color image interpolation based on incompressible Navier Stokes technique
}

\author{
C. Jittawiriyanukoon, V. Srisarkun \\ Assumption University, Samut Prakan Province, 10570, Thailand
}

\begin{tabular}{l} 
Article Info \\
\hline Article history: \\
Received Oct 7, 2019 \\
Revised Feb 6, 2020 \\
Accepted Apr 26, 2021 \\
\hline
\end{tabular}

\section{Keywords:}

Computer vision

Image analytics

Image interpolation

Masking

Navier Stokes method

Reconstruction

\begin{abstract}
Color image interpolation encompasses reconstructing parts of a video or an image based on information from the neighbor. Technique involves the restoration of noised photos and animation or image denoising. The NavierStokes (NS) technique has been widely investigated as an essential research by image restoration. These NS equations contribute spectacular results for producing an animation as they augment reality. They can boost real-time video games to be more sensible than ever. In this paper, we present the Incompressible NS approach (INS) for color image interpolating. The method per se is based on fluid flow concept to circulate directed lines from the peripheral into the area to be interpolated. The image intensity represents stream function in a computational flow of 2D fluid dynamics. The algorithm is implemented to carry on lines regarding gradient vectors at the edge of the interpolating region. It uses the improvement of powerful numerical analysis. It is also proven as an innovative idea for easing problems in image analytics as well as computer vision.
\end{abstract}

This is an open access article under the CC BY-SA license.

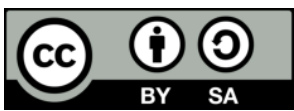

\section{Corresponding Author:}

C. Jittawiriyanukoon

Assumption University

Samut Prakan Province, 10570, Thailand

Email: pct2526@yahoo.com

\section{INTRODUCTION}

The research on dynamic flows [1] plays a noticeable role in a broad range of computer applications. The automobile industries, game companies, and space studies are regularly in need for an animation model of the 3D graphics to simulate video game and image restoration that can experience the adversity of reality. As a human, the investigation of image processing can bring substantial enhancements to existing computer graphic systems. For example, the reconstruction and denoising of underlying damaged images introduced by [2] use the recursive shrinking threshold algorithm, then the image is reconstructed by the gradient vector method. This approach is attentive for X-ray luminescence tomography industries as it identifies the dissemination of nanophosphors in object images. Above all, we spend most of the time delimited by thermo dynamic flows which agitate human activities regularly. Over the past few years, computational fluid dynamics (CFD) model [3] arises from the dynamic flows concept to examine the performance of streams. It reconstructs mathematically the progression of unsteady or steady streams from the originality. The experimental fluid dynamics (EFD) model [4] detects 3D annotations of each element of the line direction.

Digital interpolation algorithm [5] has extended presentations in photo reconstruction, image restoration, and object zooming. It brings up the use of algorithm to discard degraded fragments of object images. In computer vision [6], we kick off with a visual image or an array of them. They are image systems such as biomedical images, satellite images or artistic paintings. Most digital devices are equipped with computer vision and image processing algorithms [7]. A basic maneuver in image processing includes the 
filtering of image's intensity. It estimates the 1st order derivative filters for animation or border detection, and the 2nd order or higher for characteristics collection. For animation control and shape detection [8], the approximation of gradient descends [9] of the intensity is critical. It can result that the calculation of both magnitude of gradient vectors and its orientation is invalid. Then, option must be selected based on the orthogonality of gradient vectors. Therefore, it is confirmed that the direction of the gradient descends is more vital than the magnitude. The filtering technique is based on finite values using convolution optimization [10]-[12] regarding the presence of noise in the image or numerical model assumption.

The fundamental impression for most of the interpolating methods is to perform a refined dissemination of the information in the area encompassing the interpolating region and inpainting curvatures in an accurate manner. K. V. Madhavi et al. [13], authors propose to reconstruct the missing information using correlation between the missing block and its surroundings. In an associated article [14], the idea is magnified to guarantee that the image segmentation is applied for a combination of curvatures in order to restore the image. O. O. Akanni, et al. [15], a relation between the NS equations and the isophote (level line) direction of the image is presented and authors propose to simulate a propagation of carbonate matrix to seal the interpolating domain. Another connected article is introduced in [16]. Authors minimize the divergence to develop optical flow functions.

The article in [17] motivates a sophisticated technique to denoise based on total variation (TV) approach and discretization. The inpainting algorithm in [18] is restricted to forming straight level-lines, easily discontinued from the hole edge, and is evolved for a small hole. Note that it is to cultivate a model that allows inpainting of isophotes through large gaps, in which linking with straight connections is unattractive for modest images although direct lines provide obviously useful results for small holes. To find a continuation and smooth interpolation of level lines, the technique based on high-order partial differential equations (PDE) is presented by M. C. Leseduarte and R. Quintanilla [19]. An estimation approach to the fragmental derivation [20] using finite integration with the quadratic interpolation polynomial in PDE is also given in [21]. W. Zhou et al. [22] maintain the concept of executing a smooth continuation of the arrival angle of the isophotes. On the other hand, reflection waveform inversion [23] has been suggested to form the velocity model by confining the sensitivity of full waveform regarding two ways communication.

\section{INCOMPRESSIBLE NS (INS) METHOD}

The INS approach [24] to find out the image interpolation problem is a way to impress how color image can be restored physically. Authors inpaint extended boundaries from the edge of $\Omega$, link these stretched edges, and then fill-in the region correspondingly. The concept has been scientifically verified to fabricate acceptable result, or if negative satisfied solution. The article demonstrates an algorithm intended to resolve the smoothing gradient of the color image convolution in the lines direction. The derivative design is a discontinuous estimation of the PDE [19] as follows.

$$
I c=\nabla^{\perp} I \cdot \nabla \Delta I
$$

$I$ denotes the image convolution, $\Delta$ is the Laplacian operation $\left(\partial_{x}^{2}+\partial_{y}^{2}\right)$, and $\nabla^{\perp}$ represents the vertical gradient $\left(-\partial_{y}, \partial_{x}\right)$. Image's anisotropic diffusion can be calculated by

$$
I c=v \nabla \cdot(g(|\nabla I|) \nabla I)+\nabla^{\perp} I \cdot \nabla \Delta I .
$$

The objective is to determine a steady state result from either (1) or (2), but befitting the circumstance in the intra-region that lines are in the trend of vertical gradient $\left(-\partial_{y}, \partial_{x}\right)$. It has to be analogous to curvatures of smoothing image using the convolution $(I)$, and when $v=0$ we have the solution for the interpolation as shown in (3) and (4).

$$
\nabla^{\perp} I \cdot \nabla \Delta I=0
$$

Incompressible Newtonian flow follows the NS equations,

$$
v_{c}+v \cdot \nabla v=v \Delta v-\nabla p, \nabla \cdot v=0,
$$

Where $v$ denotes the velocity vector field, $v$ represents the anisotropic diffusion, and $p$ is a scalar pressure. In two dimensional flows, the free velocity field introduces a stream function $\Psi$, where $\nabla^{\perp} \Psi=v$. Let the smoothness, $\omega=\nabla \times v$, then the smoothness-stream function can be calculated by eliminating $p$ in (4), taking the curl of (1), and applying some facts about the geometry in two dimension: 


$$
\omega_{c}+v \cdot \nabla \omega=v \Delta \omega
$$

In case of absence of the anisotropic diffusion, $v \approx 0$, the smoothness-stream function shown in (5) implies the steady state solution as follows.

$$
v \cdot \nabla \omega=\nabla^{\perp} \Psi \cdot \nabla \Delta \Psi \approx 0
$$

In image processing, the counterpart to the smoothness-stream function can be formulated as listed below.

$$
\frac{\partial w}{\partial c}+v \cdot \nabla w=v \nabla \cdot(g(|\nabla w|) \nabla w
$$

Where $w=\Delta I$, the smoothness of image convolution, and $v=\nabla^{\perp} I$, the velocity field of image convolution. The $g$ explains a boundary-preserving diffusion and deserves

$g(0)=1, \lim _{n \rightarrow \infty} g(n)=0$, and is always declining uniformly.

The image convolution, $I$, can be figured out by resolving the Poisson's formulation

$$
\Delta I=w,\left.I\right|_{\partial \Omega}=I_{0} .
$$

In case of small $v$, the aforementioned flow takes lengthy time to reach the steady state; therefore there is pseudo-steady state that accounts for the Poisson problem, so called a relaxing equation [25] shown as follows.

$$
I_{c}-\propto(w+\Delta I)=0, \propto>0,\left.I\right|_{\partial \Omega}=I_{0} .
$$

\section{EXPERIMENTAL RESULTS AND ANALYSIS}

We illustrate using deteriorated images to check the performance of the INS approach. In each image presented below, the INS equations are executed in the interpolating region. Let us begin with calculating the smoothness $\omega$ from the individual image $I$, using information from the outside to verify the edge of the smoothness. We can obtain the smoothness-stream function through an advance Euler timing, with alternative centers in space domain for the anisotropic diffusion (which helps improve edges) and the relocation. After each step we calculate the image convolution Ic by solving the Poisson formulation iteratively. The algorithm shown in Figure 1 is implemented in number of lines of Matlab code. The results shown below are achieved in seconds of CPU time of a regular Window-based PC. We work with color images and execute interpolation on two gears separately (a color image and a mask), and the results in the end. In the experiment we consider a deteriorated image as shown in figure left and the recovered result upon INS interpolation is listed in figure right. Note that the result is improved regarding denosing, edges are illuminated (artifacts disappear) and restored. However, these are examples for the interpolation, not a complete recovered metric. The image interpolation is illustrated in Figure 2. Next examples use a different masking approach to investigate whether or not the mask will affect the quality of end results of an image. We have taken an inverse mask and $r=$ radius of the interpolation process into our account. Note how the mask and variable $r$ can recover the original image. These results are illustrated in Figure 3.

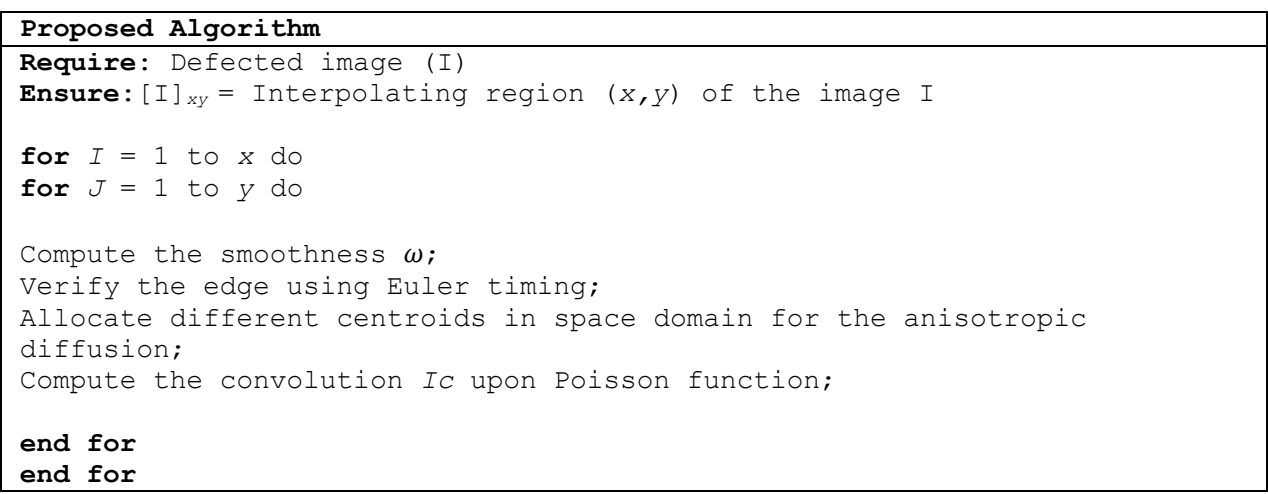

Figure 1. Proposed algorithm 

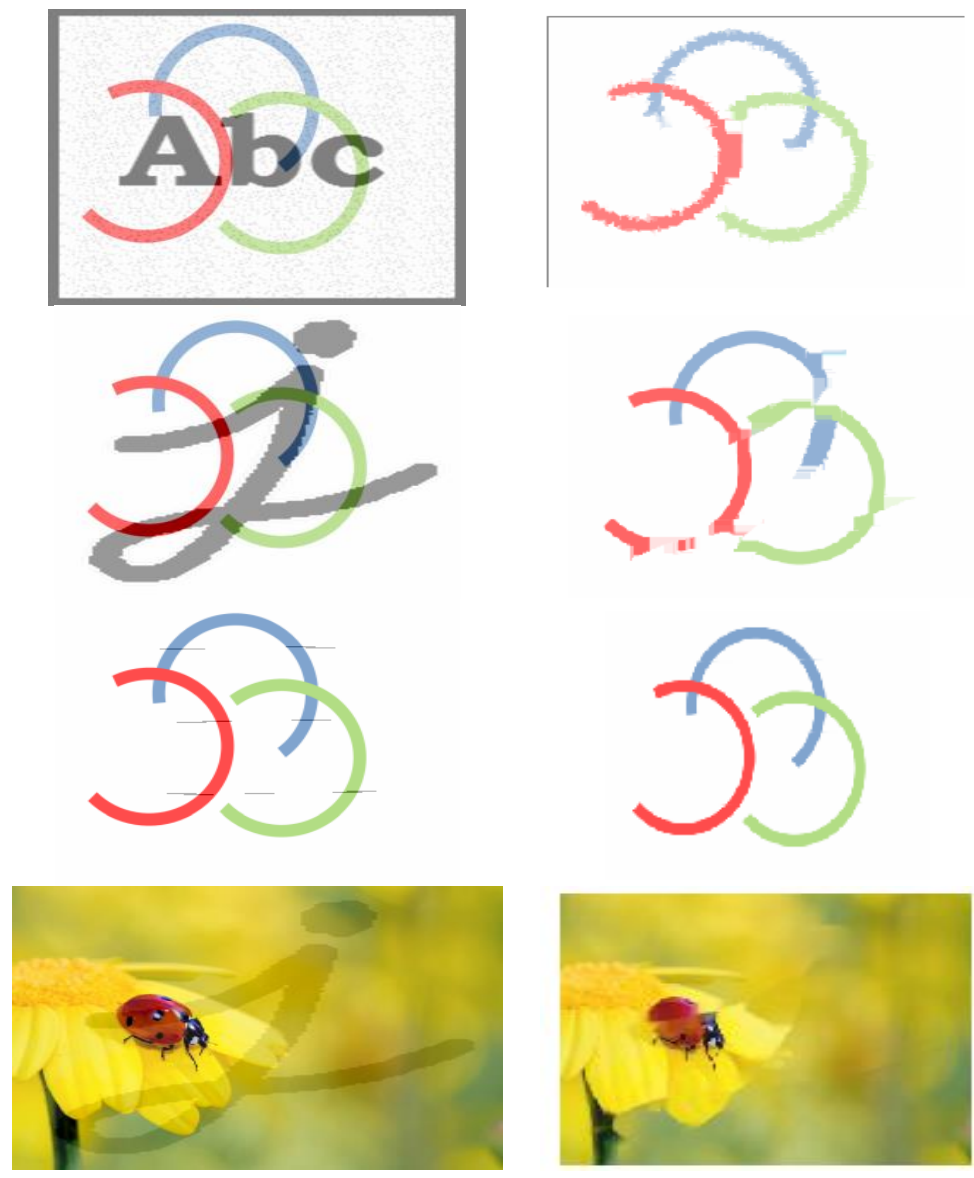

Figure 2. Deteriorated images (left) and their associated recovered images (right)
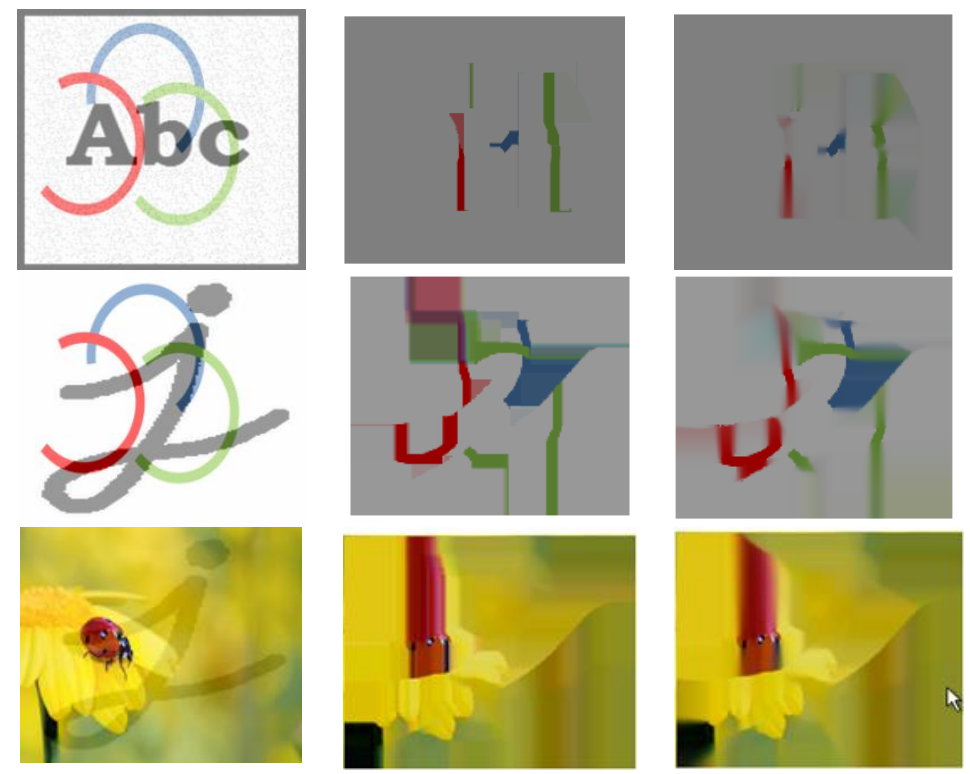

Figure 3. Deteriorated images (left) and their recovered images using inverse mask with $r=0.3$ (middle), and $r=3$ (right) 


\section{CONCLUSION}

The work evaluates the application of INS approach for color image recovery. The used technique in this article leverages an image convolution and transforms a stable input from deteriorated image to a recovered output. Comparisons are also conducted for an inverse masking scheme with the INS algorithm. Image recovery is executed by varying radius parameter into an account during the process. The experiment presented in this article confirms that the INS will not applicable to the specific damaged images. The different levels of noise degrade an original image that cannot always be perfectly recovered although it is anticipated that a state-of-the-art method like INS would complete comparatively well. Rather, this method does not provide the optimized solution for some image recovery experiments. Future work includes the investigation how to determine the degree of noise for limiting deterioration and the implementation of a practical image recovery algorithm.

\section{REFERENCES}

[1] V. S. Galkin and S. V. Rusakov, "Status of the Navier-Stokes Equations in Gas Dynamics. A Review," Fluid Dynamics, vol. 53, no. 1, pp. 152-168, 2018, doi: 10.1134/S0015462818010056.

[2] T. Liu, et al., "Regularized reconstruction based on joint L1 and total variation for sparse-view cone-beam X-ray luminescence computed tomography," Biomedical Optics Express, vol. 10, no. 1, pp. 1-17, 2019, doi: 10.1364/BOE.10.000001.

[3] P. D. Morris, et al., "Computational fluid dynamics modelling in cardiovascular medicine," Heart, vol. 102, no. 1, pp.18-28, 2016, doi: 10.1136/heartjnl-2015-308044.

[4] R. B. Salah, O. Alata, B. Tremblais, L. Thomas, and L. David, "Tomographic Reconstruction of 3D Objects Using Marked Point Process Framework," Journal of Mathematical Imaging and Vision, vol. 60, no. 7, pp. 1132-1149, 2018, doi: 10.1007/s10851-018-0800-6.

[5] L. Dai, D. H. Jiang, B. Ding, and J. K. Hahn, "Improved Digital Image Restoration Algorithm Based on Criminisi," Journal of Digital Information Management, vol. 14, no. 5, pp. 302-310, 2016.

[6] N. S. Parameswaran and D. Venkataraman, "A computer vision based image processing system for depression detection among students for counseling," Indonesian Journal of Electrical Engineering and Computer Science, vol. 14, no. 1, pp, 503-512, 2019, doi: 10.11591/ijeecs.v14.i1.pp503-512.

[7] F. Mokhtar, R. Ngadiran, T. Basheer, and A. N. A. Rahim, "Analysis of wavelet-based full reference image quality assessment algorithm," Bulletin of Electrical Engineering and Informatics, vol. 8, no. 2, pp. 527-532, 2019, doi: 10.11591/eei.v8i2.1404.

[8] A. S. A. Salam, M. N. M. Isa, and M. I. Ahmad, "M7 subtype leukemic cell edge detection techniques with threshold value comparison and noise filters," Indonesian Journal of Electrical Engineering and Computer Science, vol. 13, no. 3, pp. 1294-1302. 2019, doi: 10.11591/ijeecs.v13.i3.pp1294-1302.

[9] Z. Saringat, A. Mustapha, R. R. Saedudin, N. A. Samsudin, "Comparative analysis of classification algorithms for chronic kidney disease diagnosis," Bulletin of Electrical Engineering and Informatics, vol. 8, no. 4, pp. 1496-1501, 2019, doi: 10.11591/eei.v8i4.1621.

[10] S. Shindo, T. Goto, T. Kirishima, and K. Tsuchida, "An optimization of facial feature point detection program by using several types of convolutional neural network," Indonesian Journal of Electrical Engineering and Computer Science, vol. 16, no. 2, pp. 827-834, 2019, doi: 10.11591/ijeecs.v16.i2.pp827-834.

[11] B. S. Lazarov and O. Sigmund, "Filters in topology optimization based on Helmholtz-type differential equations," International Journal for Numerical Methods in Engineering, vol. 8, no. 6, pp. 765-781, 2011, doi: 10.1002/nme.3072.

[12] B. Bourdin, "Filters in topology optimization," International Journal for Numerical Methods in Engineering, vol. 50, no. 9, pp. 2143-2158, 2001, doi: 10.1002/nme.116.

[13] K. V. Madhavi, R. Tamilkodi, and K. Jayasudha, "Restoration of Lost Image Blocks in Wireless Transmission \& Compression via Structure and Texture Filling-in," International Journal of Advanced Research in Computer Science and Electronics Engineering, vol. 1, issue 7, pp. 131-135, 2012, doi: 10.1002/nme.116.

[14] H. Ying, L. Kai, Y. Huang, and Y. Ming, "An Improved Image Inpainting Algorithm Based on Image Segmentation," Procedia Computer Science, vol. 107, pp. 796-801, 2017.

[15] O. O. Akanni, H. A. Nasr-El-Din, and D. Gusain, "A Computational Navier-Stokes Fluid-Dynamics-Simulation Study of Wormhole Propagation in Carbonate-Matrix Acidizing and Analysis of Factors Influencing the Dissolution Process," SPE Journal, vol. 22, no. 6, pp. 2049-2066, 2017, doi: 10.2118/187962-PA.

[16] B. Kanberoglu, et al., "An Optical Flow-Based Approach for Minimally Divergent Velocimetry Data Interpolation," International Journal of Biomedical Imaging, vol. 2019, no. 2, pp. 1-14, 2019, doi: $10.1155 / 2019 / 9435163$.

[17] L. Xie, T. Wu, X. Chen and J. He, "A Cooperative Denoising Method Based on Total Variation and Discrete Wavelet Transform," 2018 IEEE 22nd International Conference on Computer Supported Cooperative Work in Design ((CSCWD)), Nanjing, 2018, pp. 45-49, doi: 10.1109/CSCWD.2018.8465312.

[18] S. S. Kanade and S. S. Gujare, "Comparative Study of Different Digital Inpainting Algorithms," International Journal of Electronics and Communication Engineering \& Technology, vol. 5, no. 12, pp. 258-265, 2014.

[19] M. C. Leseduarte and R. Quintanilla, "Spatial behavior in high order partial differential equations" Mathematical Methods in the Applied Sciences, vol. 41, no. 6, pp. 2480-2493, 2018, doi: 10.1002/mma.4753. 
[20] G. H. Gao, Z. Z. Sun, and H. W. Zhang, "A new fractional numerical differentiation formula to approximate the Caputo fractional derivative and its applications," Journal of Computational Physics, vol. 259, pp.33-50, 2014.

[21] Y. Xing and Y. Yan, "A higher order numerical method for time fractional partial differential equations with nonsmooth data" Journal of Computational Physics, vol. 357, pp.305-323, 2018.

[22] W. Zhou, R. Brossier, S. Operto, J. Virieux, and P. Yang, "Joint FWI for velocity model building: a real case study in the viscoacoustic approximation," SEG International Exposition and 87 $7^{\text {th }}$ Annual Meeting, pp. 1422-1427, 2017, doi: 10.1190/segam2017-17734253.1.

[23] R. Brossier, S. Operto, and J. Virieux, "Velocity model building from seismic reflection data by full waveform inversion," Geophysical Prospecting, vol. 63, pp. 354-367, 2015.

[24] N. P. Moshkin and D. Yambangwai, "On Numerical Solution of the Incompressible Navier-Stokes Equations with Static or Total Pressure Specified on Boundaries," Mathematical Problems in Engineering, vol. 2019, pp. 1-26, 2019, doi: 10.1155/2009/372703.

[25] G. R. Joldes, A. Wittek, and K. Miller, "An adaptive Dynamic Relaxation method for solving nonlinear finite element problems: Application to brain shift estimation," International Journal for Numerical Methods in Boimedical Engineering, vol. 27, no. 2, pp. 173-185, 2011, doi: 10.1002/cnm.1407. 